\title{
Implementation of a Locator-Based Route Switching Scheme for Improved Routing in Proxy Mobile IPv6
}

\author{
M. Okwori*a, E. N. Onwuka ${ }^{a}$, A. M. Aibinu ${ }^{a}$, O. C. Ugweje ${ }^{b}$ \\ Department of Telecommunications Engineering, Federal University of Technology Minna, Nigeria ${ }^{\text {a }}$ Research, \\ Education \& Curriculum, Digital Bridge Institute Abuja, Nigeria ${ }^{\mathrm{b}}$
}

\begin{abstract}
Proxy Mobile IPv6 (PMIPv6) is a promising IP mobility protocols that is being deployed in emerging wireless technologies. This however has a non-optimal packet route as a result of the triangular routing problem. This creates a bottle neck at the Local Mobility Anchor (LMA) thereby increasing packet delays. This paper presents the implementation of a locator-based route switching scheme on OPNET Modeler. The Mobility Access Gateway (MAG) and the LMA were enhanced by making them intelligent. This enables them to be able to check the position of the Corresponding Node $(\mathrm{CN})$ with respect to the Mobile Node $(\mathrm{MN})$ and also determine the available bandwidth on each link. From the checks made, a three-stage decision process is used to switch routing to the most optimal route that guarantees the best QoS. Node Models were developed for the MAG and LMA, network models were deployed and simulation tests were carried out. The results show that the developed scheme switched packets to a more optimal route according to the designed algorithm. The impact of this switching on differences between transmitted throughput at MN and the received throughput at $\mathrm{CN}$ was also evaluated. The receiver activity result shows a reduction in the bottleneck at the LMA-MAG link. The end-to-end delay results show over 50 milliseconds drop in packet delay as a result of the switching to a more optimal route. This shows that the packet delays result from the congestion at the LMA-MAG interface due to suboptimal routing.
\end{abstract}

Index Terms: PMIPv6, OPNET Modeling, MAG Model, LMA Model, Route Switching.

(C) 2014 Published by MECS Publisher. Selection and/or peer review under responsibility of the Research Association of Modern Education and Computer Science

\section{Introduction}

The introduction of wireless technology into the data networking world makes it possible for nodes to move about as they communicate. The connection of the node to the network can be either a nomadic connection or a

* Corresponding author.

E-mail address: michaelokwori@futminna.edu.ng 
mobile connection. A nomadic connection is one in which the node breaks connection to its initial network and makes connection (after an authentication and authorization process) to another network as it moves along [1]. This break in connection is undesirable, and greatly affects the performance of the network. On the other hand, mobile connection is one that seamlessly hands over the connection from one network to the other without a break in connection [2]. Mobility Management Protocols are responsible for ensuring a truly mobile network, and does this by keeping track of Mobile Nodes (MN) points of attachment to the network (known as location management) and ensuring the accurate delivery of packets as the MN moves around (handoff management) [3]. Examples of mobility protocols include mobile IP, network mobility, cellular IP, Virtual Internet Protocol (VIP), Loose Source Routing (LSR), and Proxy Mobile IP (PMIP). A comprehensive survey of these protocols in presented by Zhenkai et. al [4]. This work is based on improving route switching in PMIP which is currently been deployed in new technologies such as the 3GPP and LTE.

Proxy Mobile IP, a network based mobility management protocol, handles its mobility signaling with the aid of two network entities, the Mobility Access Gateways (MAGs) and the Local Mobility Anchor (LMA) [5]. The MAG detects nodes currently attached to it; informs the LMA, which acts as a topological anchor. The mapping between Home Network Prefixes (HNP), assigned to each node, and address of the MAG where node is currently attached is held at the LMA. The working principle of PMIPv6 is depicted in Fig 1. For each movement of the Mobile Node (MN), the new MAG authenticates the MN and then requests its HNP from the LMA in the Proxy Binding Update (PBU) sent to the LMA. The LMA authenticates the MAG and forwards the HNP for that particular MN to the MAG in the Proxy Binding Acknowledgement (PBA). The MAG advertises the HNP to the MN in the Router Advertisement and the MN then configures an address using the HNP [6].

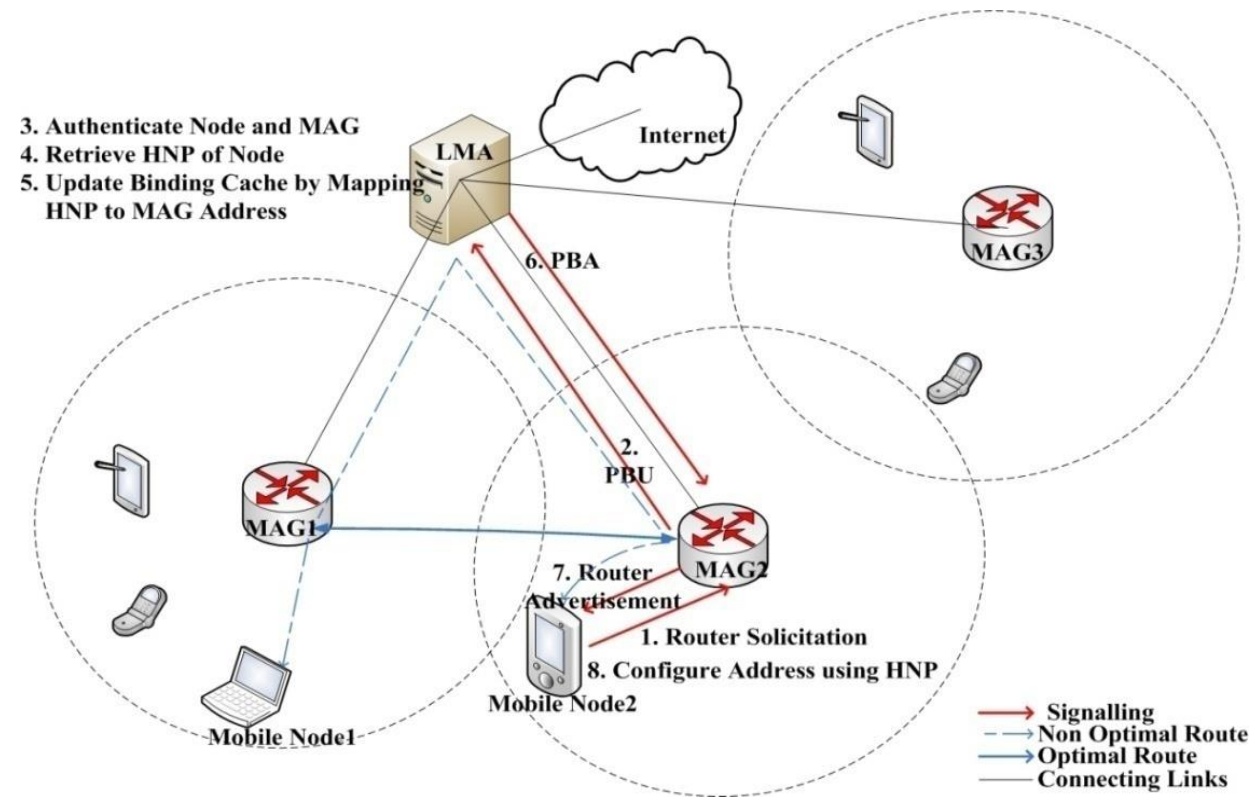

Fig. 1: System Schematic of PMIP Network

Packets sent from a node usually pass through the non-optimal route [7]. This can give rise to three shortcomings (i) a bottle neck at the LMA, (ii) inefficient bandwidth usage and (iii) increased packet delay [8]. This work demonstrates how packet routing can be enhanced on PMIPv6 networks using a MAG index based route switching scheme (MIRSS). The proposed MIRSS was implemented on OPNET Modeler 14.5 and the 
performance tested and evaluated. Similar works using Network Simulator software are presented in literature. Ref [9] carried out an implementation architecture of PMIPv6 protocol in NS-2 simulator software. While Implementation and evaluation of PMIPv6 in NS-3 Network Simulator was presented in [10]. In this work, an identifier and locator called MAG Index was assigned to all MAGs. The algorithm uses this assigned MAG Index in its decision making to switch packets routing to an optimal route.

This paper is presented in six sections. Section two surveys related works in route switching schemes in PMIPv6. Section three presents the developed OPNET models of network entities in the PMIPv6 network and shows the locator based route switching scheme deployed on the developed models. Section four presents the performance evaluation of the network models developed and the route switching scheme. Section five presents and discusses the results obtained and section six concludes the work.

\section{Review of Related Works}

A lot of research work has been done in the bid to improve the performance of proxy mobile IP based networks. Some of these works concentrated on enhancing security, reducing overhead, reducing latency and packet delays, enabling inter-domain mobility management and a lot concentrated on optimizing the routing paths of packets.

Sanjin and Myung-Ki, 2008, proposed an extension of the route optimization scheme for a node based mobility management scheme known as the Mobile IPv6 and shifted the signalling from the mobile node to the MAG. Assumption was made that the IPv4 and IPv6 address of all MAGs are known to each other by bootstrapping mechanism or out-of-band signalling method. Return Routability Test involving Proxy Care-ofAddress Test/Care-of address Test Init (Proxy CoT/CoTI) are performed in the scheme, but what actually triggers the route switching was not specified [8]. Byung-Jin et al., 2008, proposed a scheme for nodes using on-link IPv6 (ON) prefix type, where the MAG acts as the Access Router of the On-link Node. The mechanism used the MAG's routing table and security policy database to find the optimized route without involving the LMA [11]. In [12] Tsunehiko et al., 2008, also presented four different approaches to route optimization. The first approach was the LMA and MAG binding cache entry approach, in which, after the LMA receives the first packet, it sends a Correspondent Binding Update to the MAG notifying it of the destination MAG. The downside is the first packet gets routed non-optimally. Other approaches presented include the Binding Query Approach, Multicasting Binding Cache to MAGs and the Ring, Bus and Mesh-Based Approach.

Jungwook et al., 2009, made use of the "Route Optimization Process" which comprises of MIPv6 binding update and PMIPv6's proxy binding updates. A demonstration was presented of the route optimization scheme between a fixed Corresponding Node $(\mathrm{CN})$ with MIPv6 and a MAG when communication was initiated by the Mobile Node and also when communication was initiated by the CN. The work also outlined the route optimization sequence in a purely PMIP network between two MAGs. The route optimization trigger was also not specified [13]. In ref [14], Young-Hyun and Tai-Myoung, 2009, worked on eliminating the double signalling cost of Dutta's Light Weight Route Optimization [13]. This was achieved using Correspondent Binding Information (CBI) from the LMA notifying both MAGs of the possibility of direct communication. Both MAGs then exchange Correspondent Binding Update (CBU) and Correspondent Binding Acknowledgement (CBA) and the optimized route is set up both ways. Jin et al., 2009, proposed an LMA initiated RO (LIRO) protocol. There was an assumption that LMA keeps two binding cache entries, a Correspondent Binding cache Entry (CBE) and a Local Binding cache Entry (LBE). The route optimization mechanism was then described for when there was no prior optimization path and when there was a prior optimization path [15].

Jianfeng et al., 2012, presented a comprehensive survey of other route optimization schemes for Proxy Mobile IPv6 [16]. Each of these schemes was unique in either signalling used to achieve route optimization or in network entity in charge of the optimization process.

Notice that in all the route optimization schemes presented above, the trigger for switching from one route to another was not clearly defined. But this work presents a route switching scheme with a clearly defined trigger. 
We also demonstrated the development of the PMIPv6 network entities on OPNET modeler. The models developed were used to run simulations to evaluate the performance as opposed to analytical techniques used in the works presented above. A table of comparison between previous scheme and the proposed scheme is presented in Table 1.

Table 1: Comparison of route optimization schemes in Proxy Mobile IP

\begin{tabular}{|c|c|c|c|}
\hline Authors & Title & $\begin{array}{l}\text { Method of } \\
\text { Evaluation }\end{array}$ & Metric Evaluated \\
\hline Sanjin et al. (2008). & $\begin{array}{l}\text { Route Optimization Scheme for } \\
\text { Proxy Mobile IPv6 (PMIPv6). }\end{array}$ & None & None \\
\hline $\begin{array}{l}\text { Byung-Jin et al. } \\
\text { (2008). }\end{array}$ & $\begin{array}{l}\text { PMIPv6 Route Optimization } \\
\text { Mechanism using the Routing Table } \\
\text { of MAG. }\end{array}$ & Analytical & $\begin{array}{l}\text { Total cost against number of cells for various } \\
\text { velocities and densities of } \mathrm{MN} \text { in area. }\end{array}$ \\
\hline $\begin{array}{l}\text { Tsunehiko et al. } \\
\text { (2008). }\end{array}$ & $\begin{array}{l}\text { Route Optimization for Proxy Mobile } \\
\text { IPv6 in IMS Network Network }\end{array}$ & Analytical & $\begin{array}{l}\text { Delay due to route optimization against delay } \\
\text { between MAG and LMA. }\end{array}$ \\
\hline $\begin{array}{l}\text { Jungwook et al. } \\
\text { (2009). }\end{array}$ & $\begin{array}{l}\text { Route Optimization in PMIPv6 } \\
\text { Environment. }\end{array}$ & Analytical & $\begin{array}{l}\text { Total cost against number of cells for various } \\
\text { velocities and densities. }\end{array}$ \\
\hline $\begin{array}{l}\text { Young-Hyun et al. } \\
\text { (2009). }\end{array}$ & $\begin{array}{l}\text { Enhanced Light Weight Route } \\
\text { Optimization in Proxy Mobile IPv6. }\end{array}$ & Analytical & $\begin{array}{l}\text { Location update cost against number of cells for } \\
\text { particular density and velocity. }\end{array}$ \\
\hline Jin et al. (2009). & $\begin{array}{l}\text { LMA Initiated Route Optimization } \\
\text { Protocol for Improving PMIP } \\
\text { Handover Performance. }\end{array}$ & Analytical & Handover latency for MAGs against time. \\
\hline $\begin{array}{l}\text { Proposed Scheme } \\
\text { (2013) }\end{array}$ & $\begin{array}{l}\text { MAG Index based Route Switching } \\
\text { Scheme (MIRSS) }\end{array}$ & $\begin{array}{l}\text { Simulation } \\
\text { (OPNET) }\end{array}$ & $\begin{array}{l}\text { Packet delay against time, throughput difference } \\
\text { against route and percentage link utilization } \\
\text { against time. }\end{array}$ \\
\hline
\end{tabular}

\section{Development of the Algorithm and OPNET Models}

Various terms used in the development of the algorithm are defined in Table 2.

Table 2: Definition of terms

\begin{tabular}{ll}
\hline Term & Used to Represent \\
\hline$M_{M N}$ & the MAG hosting the Mobile Node (MN) \\
$M_{C N}$ & the MAG hosting the Corresponding Node (CN) \\
$M_{i_{\_} M N}$ & MAG index of the MAG hosting the MN \\
$M_{i_{-} C N}$ & MAG index of the MAG hosting the CN \\
$B_{M A G \_L M A}$ & available bandwidth on MAG to LMA link \\
$B_{M A G \_M A G}$ & available bandwidth on MAG to MAG link \\
$S_{I}$ & result of index test \\
$S_{2}$ & result of link condition test \\
$S_{\text {Final }}$ & final route result. \\
\hline
\end{tabular}

The developed MAG Indexed-based Route Switching Scheme (MIRSS) follows the five steps outlined as follows: 
Step 1. $M_{M N}$ sends MAG index request to LMA on arrival of packets from $\mathrm{MN}$. The request sent contains $\mathrm{CN}$ address.

Step 2. LMA checks the location of $\mathrm{CN}$ and sends $M_{i_{-} C N}$ to the $M_{M N}$

Step 3. $M_{M N}$ computes $S_{l}$ and $S_{2}$ using (1) and (2).

$$
\begin{aligned}
& S_{1}= \begin{cases}1 & \text { if }\left|M_{i_{-} M N}-M_{i_{-} C N}\right|=\operatorname{In}\left[\lim _{n \rightarrow \infty}\left(1+\frac{1}{n}\right)^{n}\right] \\
0 & \text { otherwise }\end{cases} \\
& S_{2}=\left\{\begin{array}{cc}
1 & \text { if } B_{\text {MAG_LMA }}-B_{\text {MAG_MAG }} \leq\left(\operatorname{In}\left[\lim _{n \rightarrow \infty}\left(1+\frac{1}{n}\right)^{n}\right]-\operatorname{In}(e)\right) \\
0 & \text { otherwise }
\end{array}\right.
\end{aligned}
$$

Step 4. $M_{M N}$ performs a logical operation on $S_{1}$ and $S_{2}$

$$
S_{\text {Final }}=S_{1} \oplus S_{2}
$$

Step 5. $M_{M N}$ switches to a direct link to $M_{C N}$ if $S_{\text {Final }}$ evaluates to true (1), but $M_{M N}$ routes packets to LMA if $S_{\text {Final }}$ evaluates to false (0).

OPNET Modeler 14.5 was used to model the locator based route switching scheme for PMIPv6 Network. The OPNET design procedure follows a three-tiered hierarchy that includes the Network Project Model, the Node Model and the Process Model. The Network Project Model represents the network topology which consists of nodes, links and subnets. The Node Model specifies object in network domain and the node's internal architecture in terms of functional elements and data flow between them. The Process Model describes the behavior of processes (algorithms, protocols, applications) specified using Finite State Machines (FSM) and extended high level language. The FSMs run on sets of programming codes referred to as PROTO-C which is combined $\mathrm{C}++$ codes and Kernel Procedures which are indigenous OPNET codes.

Node Models were developed to model the routing functions of the Mobility Access Gateway (MAG), the Local Mobility Anchor (LMA), the Mobile Node (MN) and the Corresponding Node (CN). Each of the node models had process models that were designed to carry out the functions of MAG, LMA MN and CN respectively.

\subsection{MAG Model Design}

The developed Node Model of the MAG and Process Model of the MAG_CPU are shown in Fig. 2. (a) and (b). The PKT_COUNT processor was for collecting the statistics of packets received at each MAG. The Node Model of the MAG was developed to have a wireless interface for communicating with MNs, three wired interfaces connecting to the LMA and its two neighboring MAGs. It accepts packets from the MN, processes it and sends it out of one of the wired interfaces. 


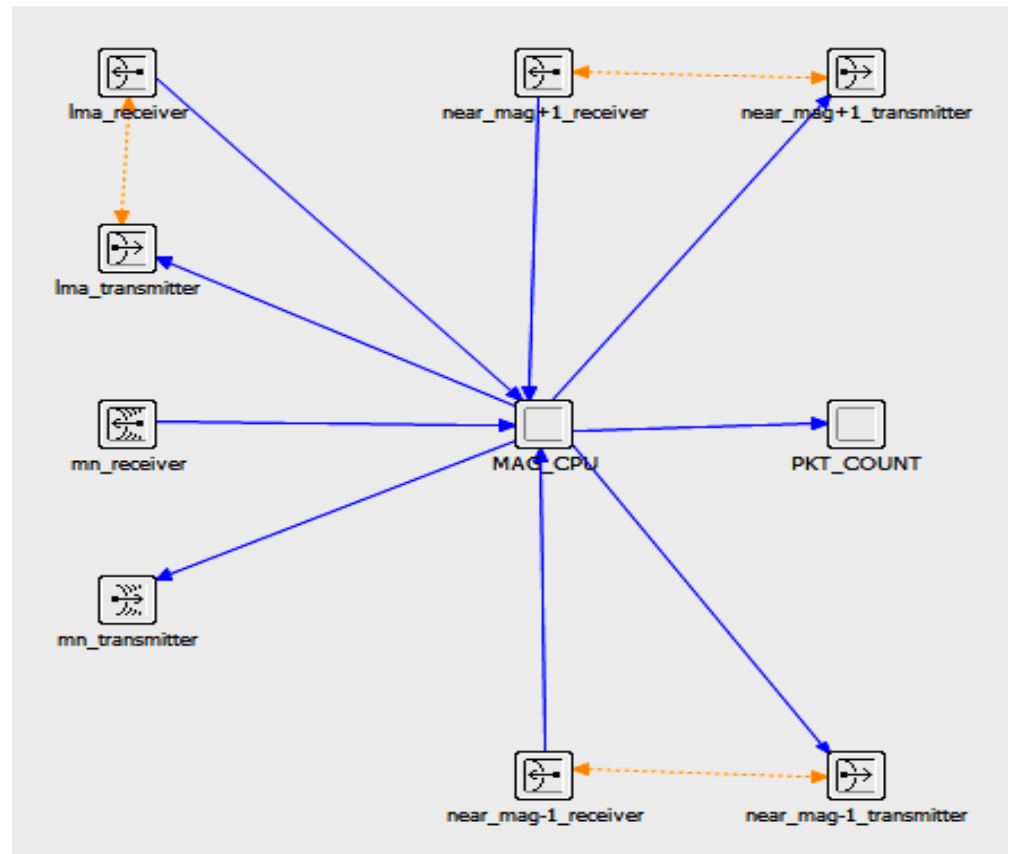

Fig. 2. (a) Node Model Design of MAG

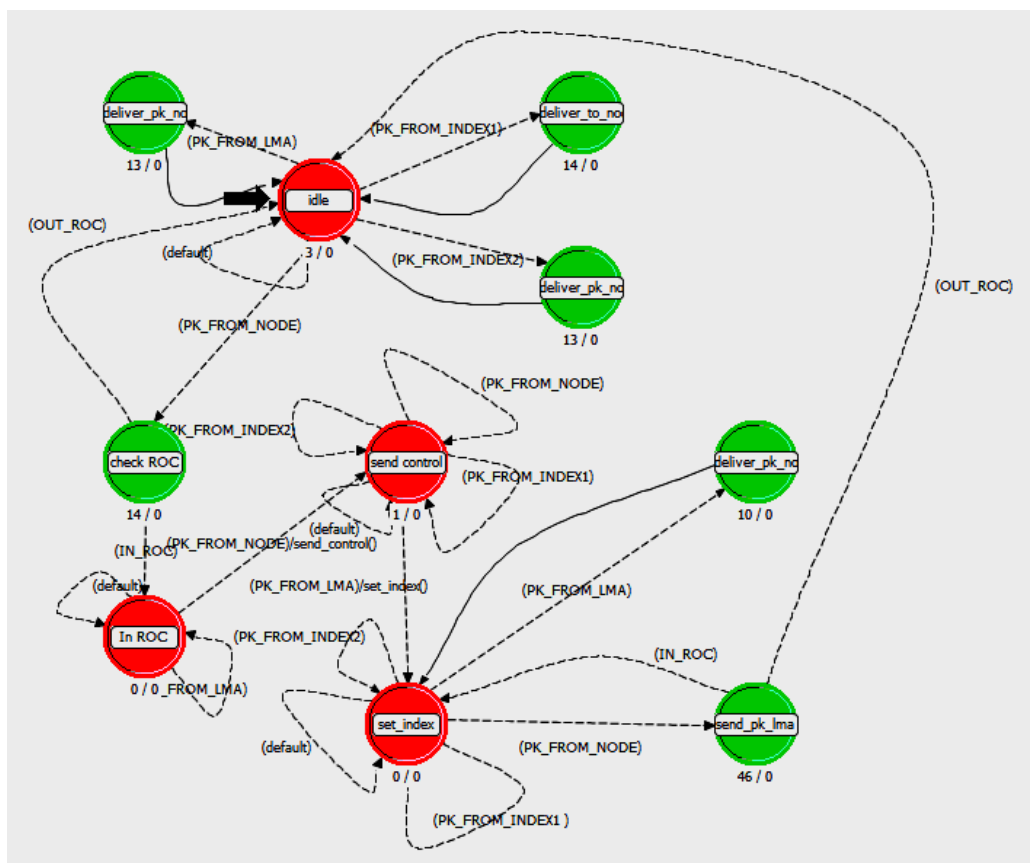

Fig. 2. (b) Developed FSM for MAG_CPU 
The process model was designed using ten states, four forced states and six unforced states. The init state termed idle initializes the variables used in the various other states and can transit to four other states depending on the transition condition that evaluates to true. If a packet arrives from the $\mathrm{MN}$ via the radio interface, transition condition $P K_{-} F R O M \_N O D E$ evaluates to true and it transits to state check $R O C$ where the distance of the node from the MAG is measured to get the relative signal strength of the received packet. If the $\mathrm{MN}$ is within the region of coverage of the MAG (this was set to be $10 \mathrm{~km}$ ), transition condition $I N \_R O C$ evaluates to true and a transition to state send control occurs. This state is responsible for sending the MAG index request to the LMA and does this using a transition executive send control. On getting the MAG Index response from the LMA, it uses transition executive set index to assign the received Index to a State variable and transits to set_index state. Any subsequent packet from MN is delivered to the LMA by the send_pk_lma state. All the five deliver_pk_node states are responsible for delivering packets to the MNs attached to the MAG. Proto-C codes were written for each of these states and transition executives to carry out the described tasks.

\subsection{LMA Model Design}

The developed Node Model of the LMA and Process Model of the LMA_CPU are shown in Figs. 3. (a) and (b).

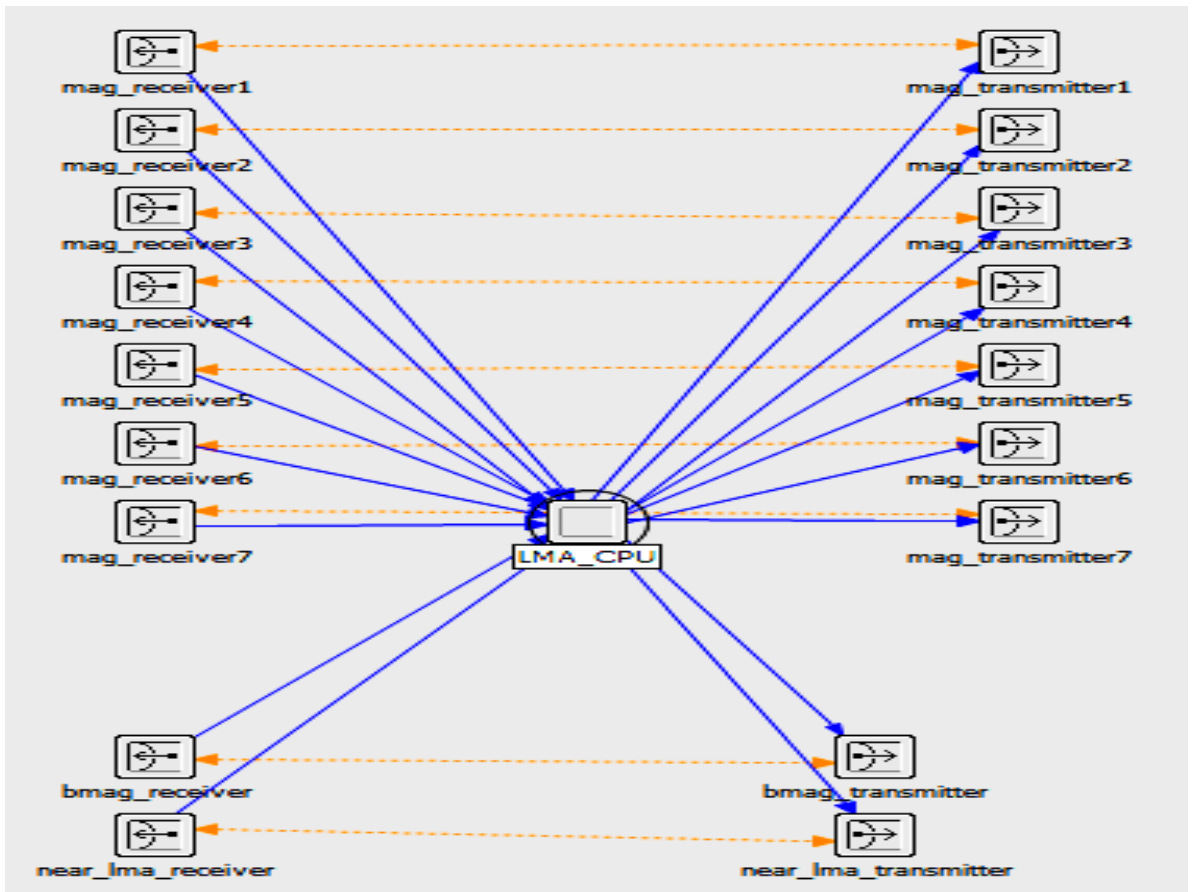

Fig. 3. (a) Node Model Design of LMA 


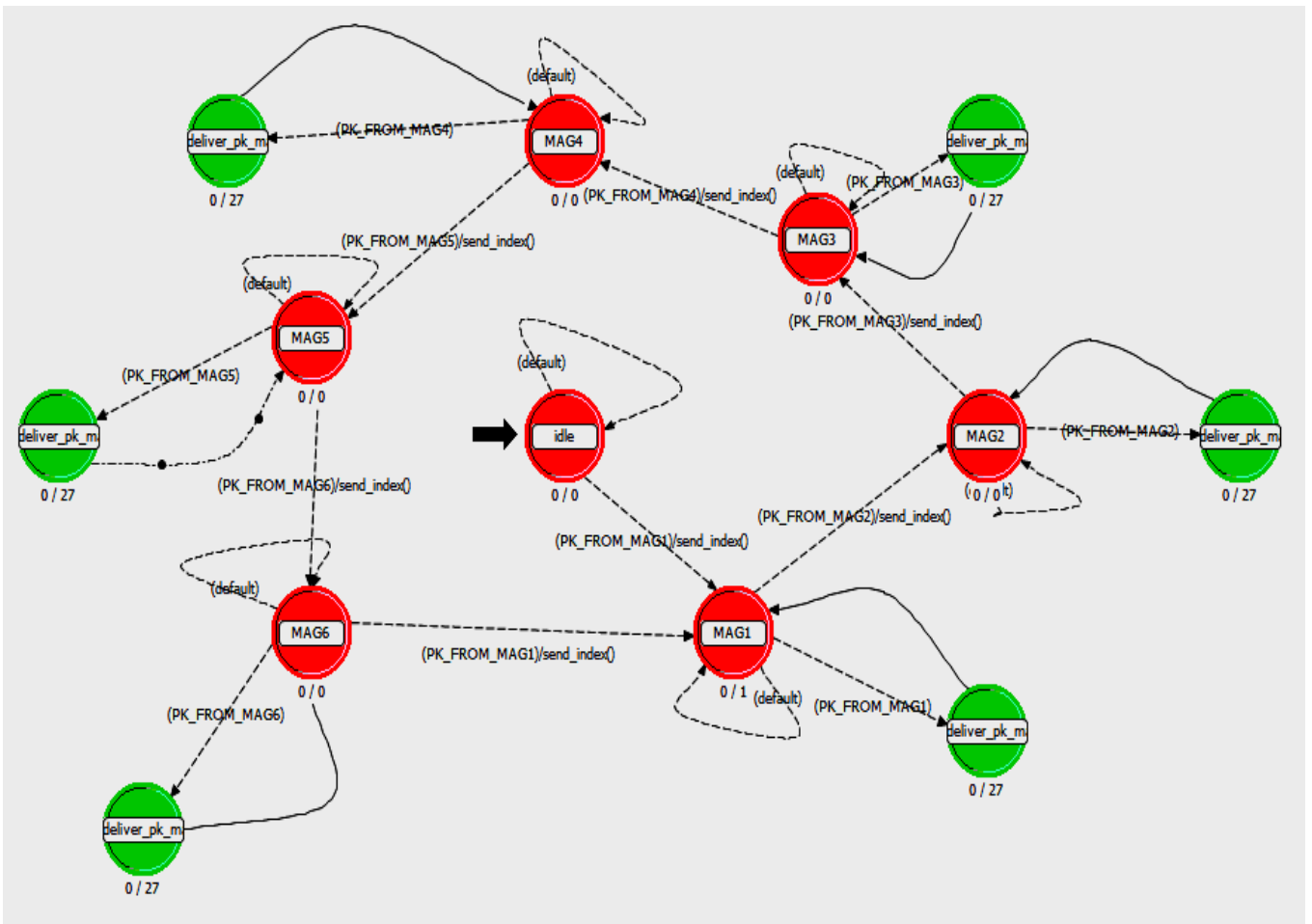

Fig. 3. (b) Developed FSM for LMA_CPU

The node model was developed to have the capability of interfacing with seven different MAGs via wired interfaces, holds the Binding Cache Entry of all MN in its domain and the details of all the MAGs (both the identifier and the introduced locator). The bmag and near_lma interfaces are for a future work and were not utilized in this particular work.

The process model of the LMA_CPU has thirteen states, each of the six MAGs in the network model has two states each (one forced and one unforced) and the init state called idle. The first packet from the MAG makes PK_FROM_MAG to evaluate to true and the transition executive send_control returns the MAG index response to the MAG. Subsequent packets are delivered using the deliver_pk_mag state. Proto-C codes were written for each of these states to carry out the described tasks.

\subsection{MN and CN Model Design}

Two node models were developed, one for the $\mathrm{MN}$ and the other for the $\mathrm{CN}$ tethered at a particular MAG. These were made to be able to generate packets, send, and receive transmitted packets, and also measure the end-to-end packet delays of received packets. These are shown in Fig 4. The process model of the MN and CN were designed to be simple and to achieve the minimal functionalities desired. That for the MN has three processors $M N \_C P U$, data_gen, and comm_setup, while the $\mathrm{CN}$ has one processor sink. 


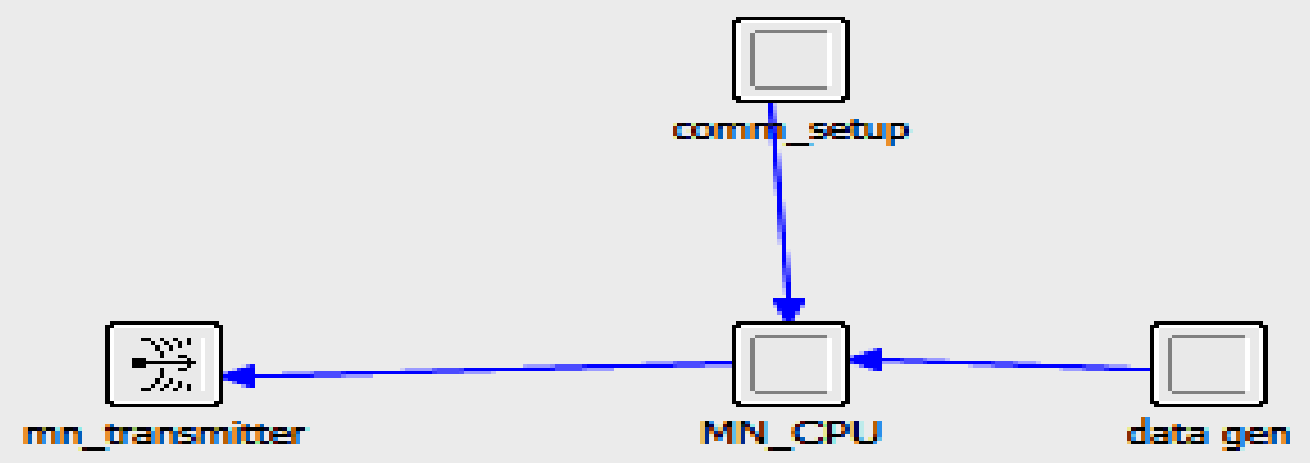

Fig. 4. (a) Node Model Design of MN

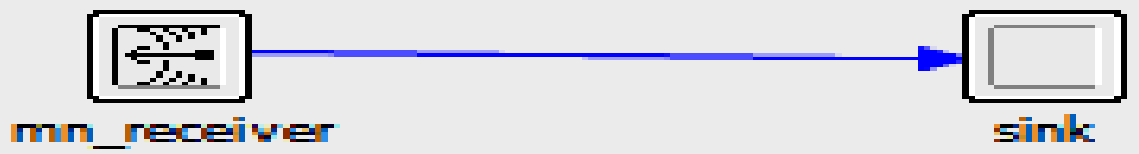

Fig. 4. (b) Node Model Design of CN

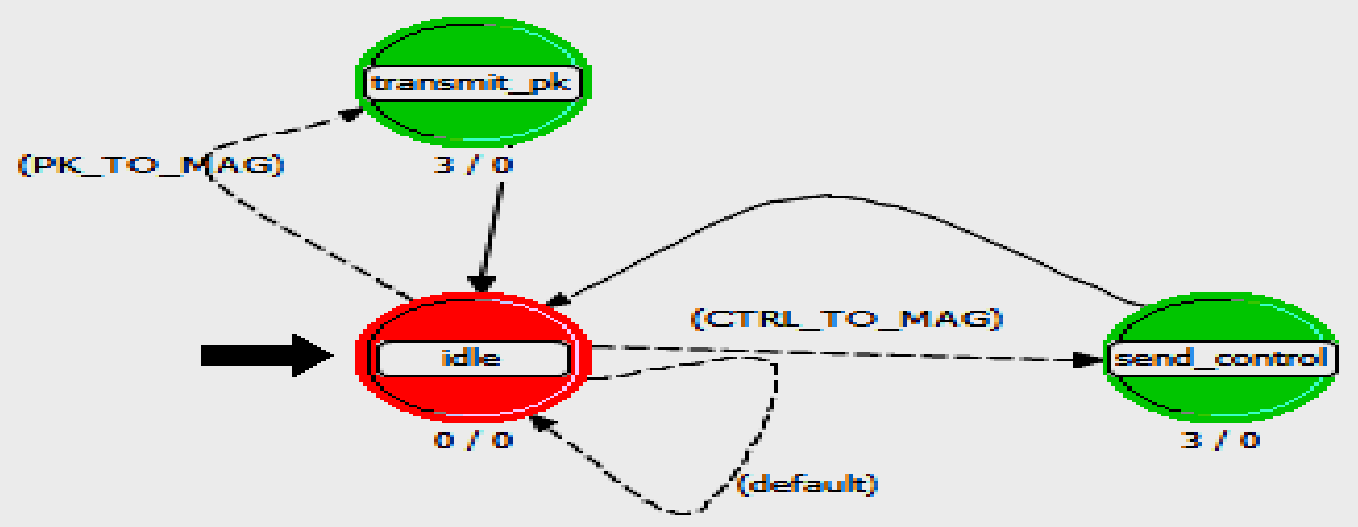

Fig. 4. (c) Developed FSM for MN_CPU 


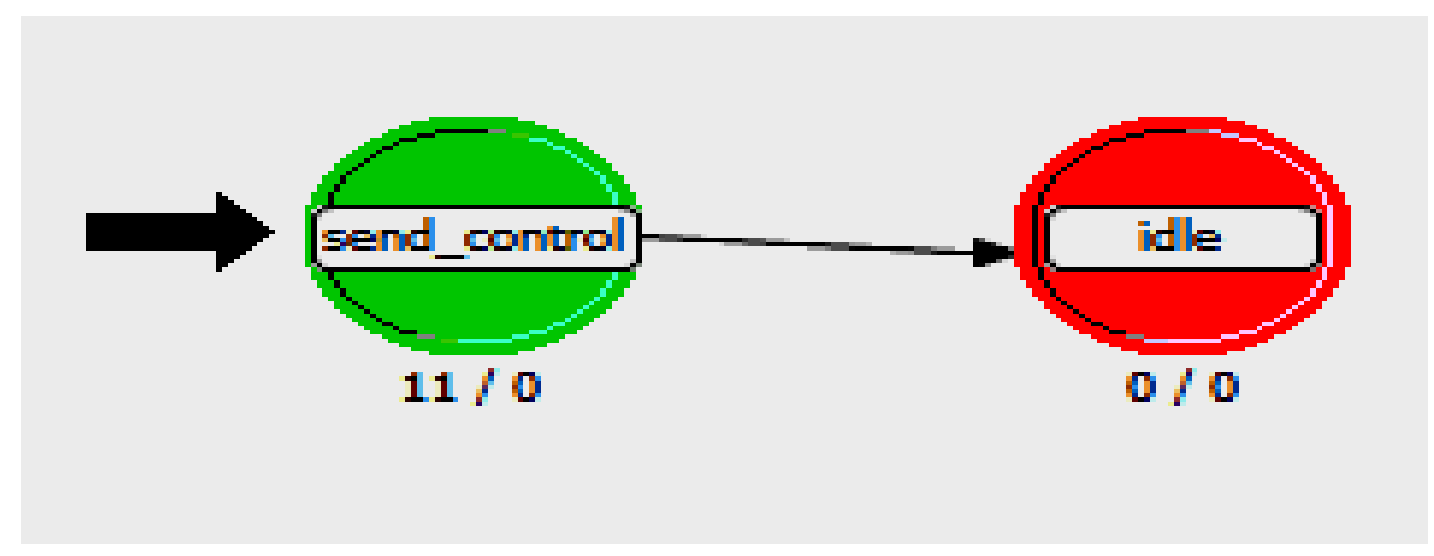

Fig. 4. (d) Developed FSM for comm_setup

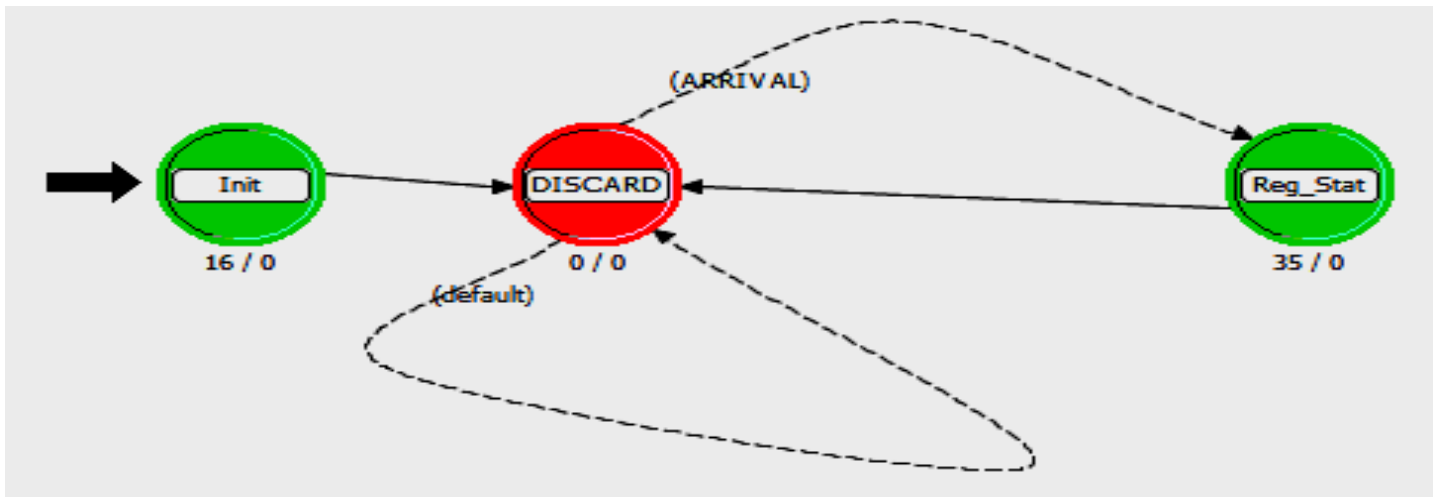

Fig. 4. (e) Developed FSM for CN_Sink

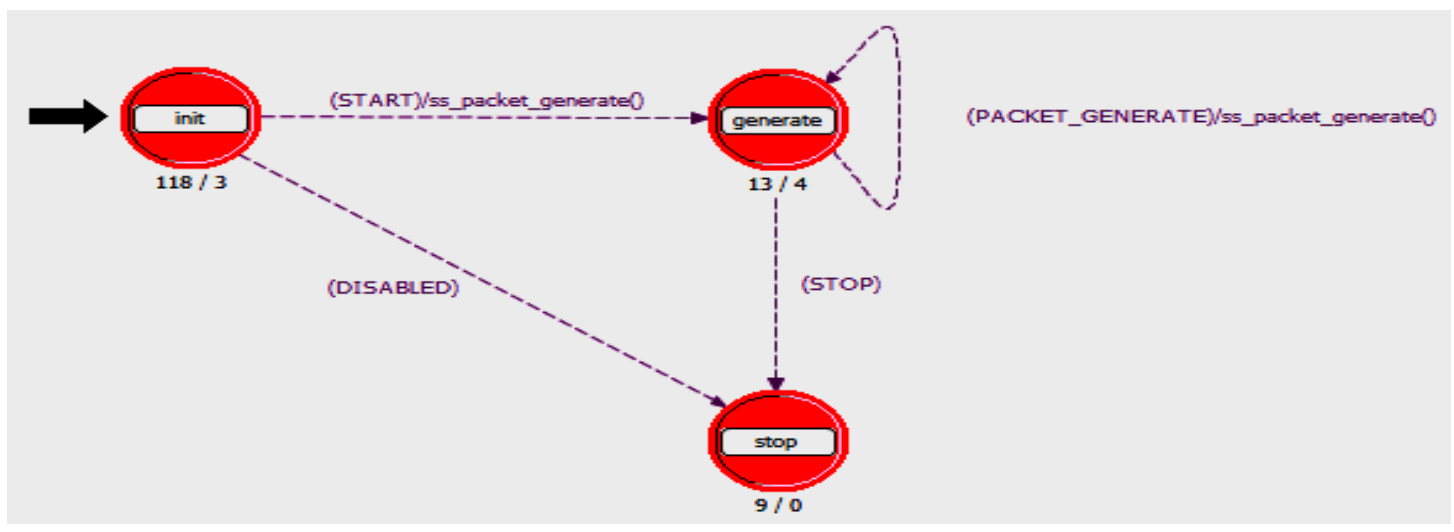

Fig. 4. (f) FSM for data_gen 
The FSM for the $M N \_C P U$ in Fig. 4. (c) is responsible for coordinating the activities of the MN and was implemented using three states. The initial state is the idle state, and the program flow waits at this state till either of its transition conditions evaluates to true. A packet arriving from the data_gen process triggers a transition to transmit_p $k$ while a packet arriving from the comm._setup process causes transition to end_control state. The FSM in Fig. 4. (d) for the comm._setup process is responsible for sending a control signal containing the address of the node it wants to communicate with during the communication setup process. The FSM in Fig. 4. (f) is from the OPNET's process for a simple source and was used to generate packets that were sent to the CN. Fig. 4. (e) is the FSM for the sink in the CN and was implemented using the OPNET's FSM for a sink. It is responsible for receiving packets sent to the $\mathrm{CN}$ and registering statistics (e.g. end-to-end delay, packet size). Proto-C codes were written for all these states.

\section{Simulation Setup}

Two network models were designed to test the performance of the developed scheme and are shown in Figs. 5. (a) and (b). The first network model had the LMA situated in the middle while the MAGs were distributed around it. This deployment scheme keeps to a minimum the distance of the MAGs from the LMA. The mean distance of the LMA from each MAG was set at $35 \mathrm{~km}$. In practice this may not always be possible, therefore the second network model positioned the LMA at varying distances from the MAGs. In this network model, the closest MAG to the LMA was set at $20 \mathrm{~km}$ while the farthest was at $60 \mathrm{~km}$. This second model checks the impact of great distance between LMA and MAGs on the performance of the designed scheme.

Two scenarios were run on both network models to test the sensitivity of the designed scheme to changes in available bandwidth. In scenario 1 the MAG to MAG links were assigned more available bandwidth than the MAG to LMA links while in scenario 2, the assigned available bandwidth on the MAG to LMA links were more than the MAG to MAG links.

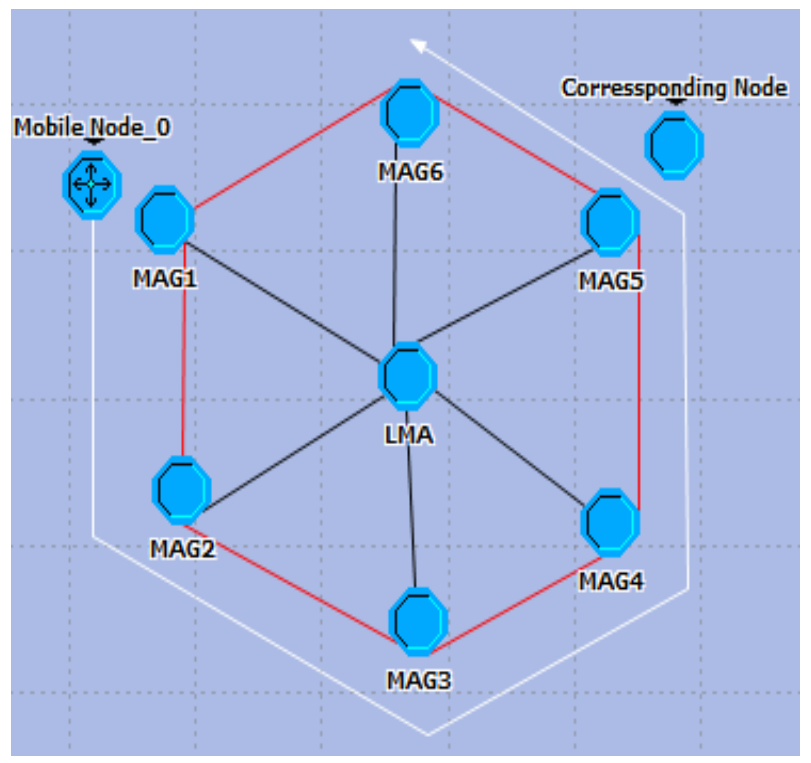

Fig. 5. (a) Network Model_1 with LMA at Edge of MAGs 


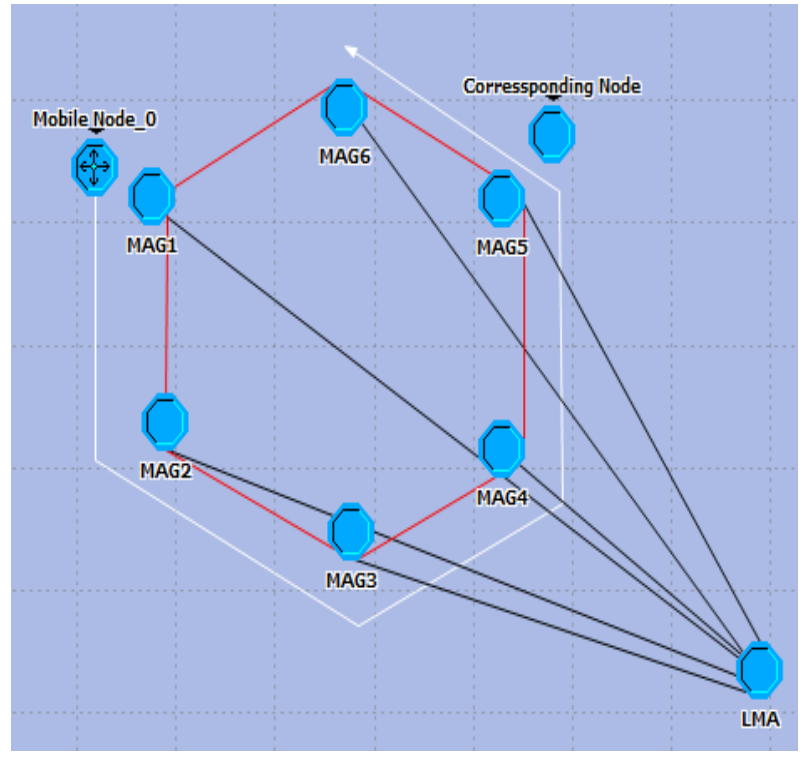

Fig. 5. (b) Network Model_2 with LMA at Centre of MAGs

\section{Results and Discussion}

The results collected were the packet count on all the interfaces of the MAGs, throughput of packets sent by $\mathrm{MN}$ and throughput of packets received by $\mathrm{CN}$, end-to-end packet delays and activity of the 3 wired interfaces of MAG5 where $\mathrm{CN}$ was tethered. The throughput used in this paper is as defined by the OPNET simulator and represents the average number of packets successfully received or transmitted by the receiver or transmitter channel per second. The packet count on the MAGs' interfaces was monitored to detect when the scheme switched routing to a more optimal route and shown on Table 3 and Table 4 for both scenario 1 and scenario 2 respectively. A comparison of the throughput at $\mathrm{MN}$ and throughput at $\mathrm{CN}$ in Fig. 6 shows the impact of the designed scheme on differences in transmitted throughput at $\mathrm{MN}$ and received throughput at $\mathrm{CN}$ for each of the various routes. The end-to-end delay results in Fig. 9. show drop in packet delay as a result of the switching to a more optimal route. Finally the receiver activity in Fig. 7. shows the distribution of load at the corresponding MAG interfaces and LMA interfaces which reflects a reduction in the bottleneck at the LMA to MAG link.

Table 3: Number of Packets Sent through each MAG Interface for Scenerio1

\begin{tabular}{l|l|c|c|c}
\hline \multirow{2}{*}{ MAG No. } & \multicolumn{2}{|c|}{$\begin{array}{c}\text { Number of Packets Sent through MAG and LMA } \\
\text { Interfaces }\end{array}$} & $\begin{array}{c}\text { Route } \\
\text { Switched? }\end{array}$ \\
\cline { 2 - 4 } & LMA Interface & $\begin{array}{c}- \text { MAG } \\
\text { Interface }\end{array}$ & + MAG Interface & \\
\hline 1 & 439 & 0 & 0 & No \\
2 & 771 & 0 & 0 & No \\
3 & 1307 & 0 & 0 & No \\
4 & 1 & 0 & 1260 & Yes \\
5 & 1 & 0 & 0 & Yes \\
6 & 1 & 776 & 0 & Yes \\
\hline
\end{tabular}


Table 4: Number of Packets sent through each MAG Interface for Scenario 2

\begin{tabular}{l|l|c|c|c}
\hline \multirow{2}{*}{ MAG No. } & \multicolumn{2}{|c|}{ Number of Packets Sent through MAG and LMA } & \multirow{2}{*}{$\begin{array}{c}\text { Route } \\
\text { Interfaces }\end{array}$} \\
\cline { 2 - 4 } & LMA Interface & $\begin{array}{c}- \text { MAG } \\
\text { Interface }\end{array}$ & + MAG Interface & \\
\hline 1 & 439 & 0 & 0 & No \\
2 & 771 & 0 & 0 & No \\
3 & 1307 & 0 & 0 & No \\
4 & 1 & 0 & 1260 & Yes \\
5 & 1 & 0 & 0 & Yes \\
6 & 1 & 776 & 0 & Yes \\
\hline
\end{tabular}

A total of 6315 packets were generated during the one hour simulation time. From Table 3 for scenario 1, it shows that when the $\mathrm{MN}$ is in the region of coverage (ROC) of $M A G_{1}, M G_{2}$ and $M A G_{3}$, the route taken by the packets were the MAG to LMA link. The 1 packet sent by $\mathrm{MAG}_{4}, \mathrm{MAG}_{5}$ and $\mathrm{MAG}_{6}$ on the $\mathrm{LMA}$ interface is the MAG Index Request, after which the packet forwarding is switched to the MAG-to-MAG link by both $\mathrm{MAG}_{4}$ and $\mathrm{MAG}_{6} . \mathrm{MAG}_{5}$ is where the $\mathrm{CN}$ is hosted, therefore packets are forwarded directly to it on the wireless interface after the MAG index request is sent to the LMA on the LMA interface.

Table 4 shows similar results to the first scenario except that packet switching does not occur when MN gets to $\mathrm{MAG}_{4}$ and $\mathrm{MAG}_{6}$. This is as a result of the link condition test in equation (2) evaluating to 0 making the final route test to evaluate to false.

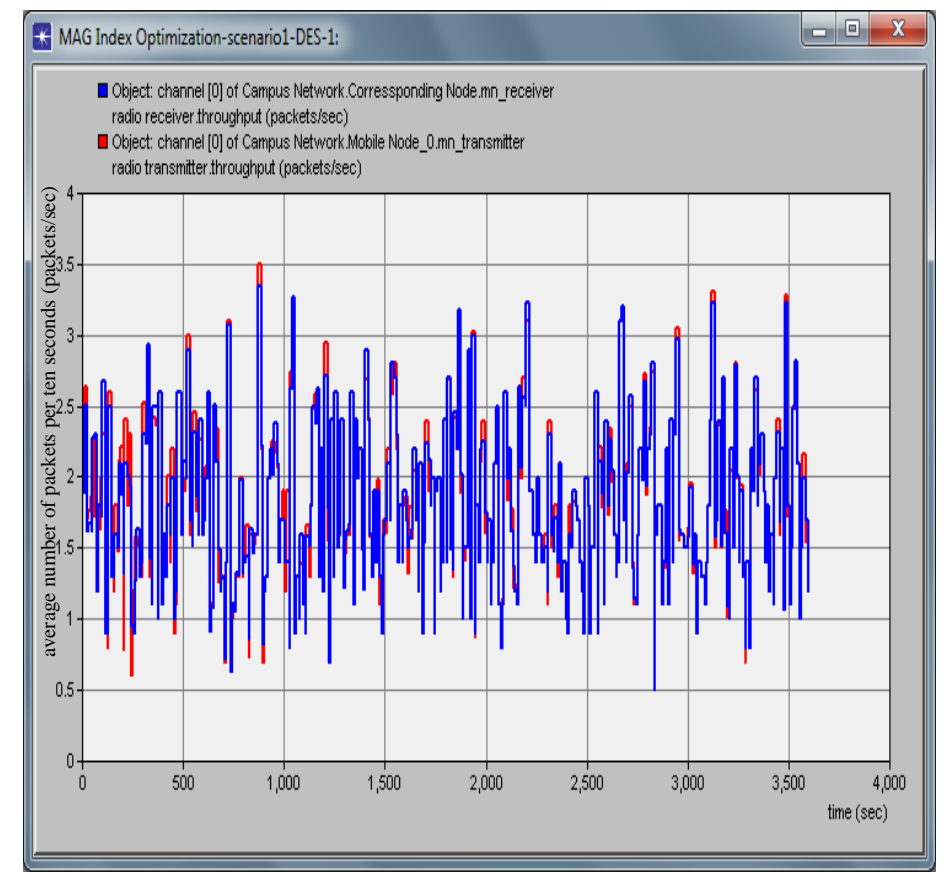

Fig. 6. Overlay of Mobile Node and Corresponding Node Throughputs 


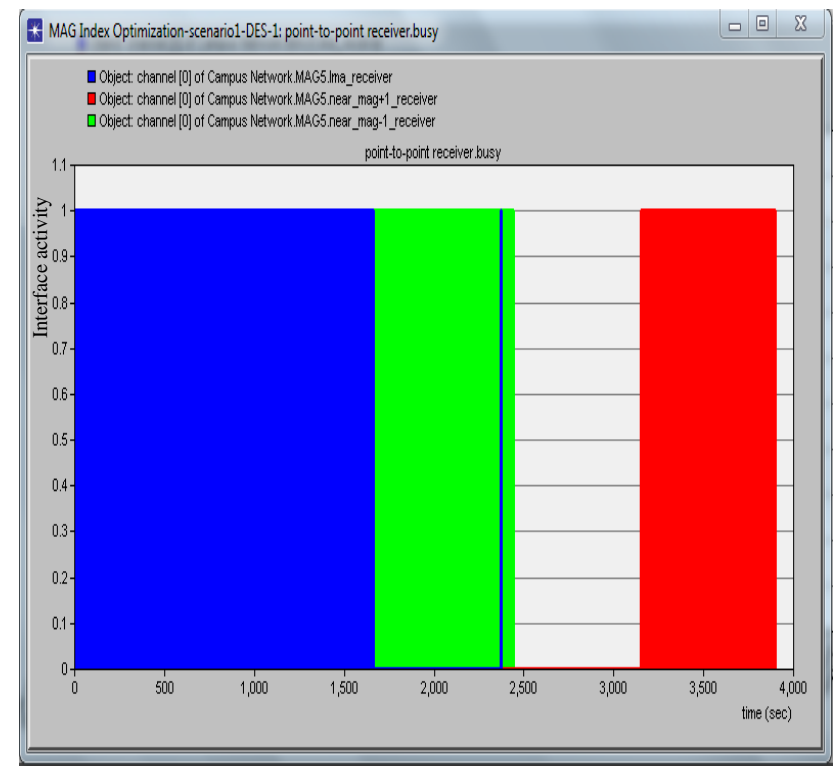

Fig. 7. MAG5 Receiver Activity

Fig. 6 shows an overlay of throughput of packets sent from the MN on the throughput of packets received by the $\mathrm{CN}$. Each of the spikes represents duration of ten seconds and the corresponding average number of packets sent/received per 10 seconds plotted against simulation time. It can be seen that there are slight differences in throughput values; this is as a result of delays experienced by packet resulted in their arriving outside the ten second time-window used to collect the results. These differences in throughput values were measured and the maximum absolute deviation and the Mean Square deviation from the throughput at the MN for each of the various routes plotted in Fig. 8. (a) and (b).

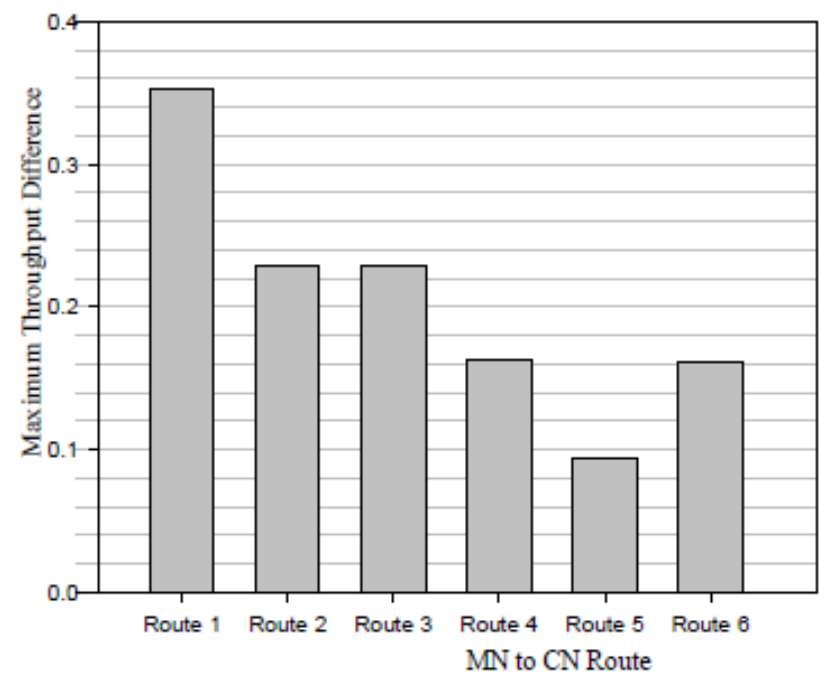

Fig. 8. (a) Maximum difference in Transmitted Throughput and Received throughput for Routes 


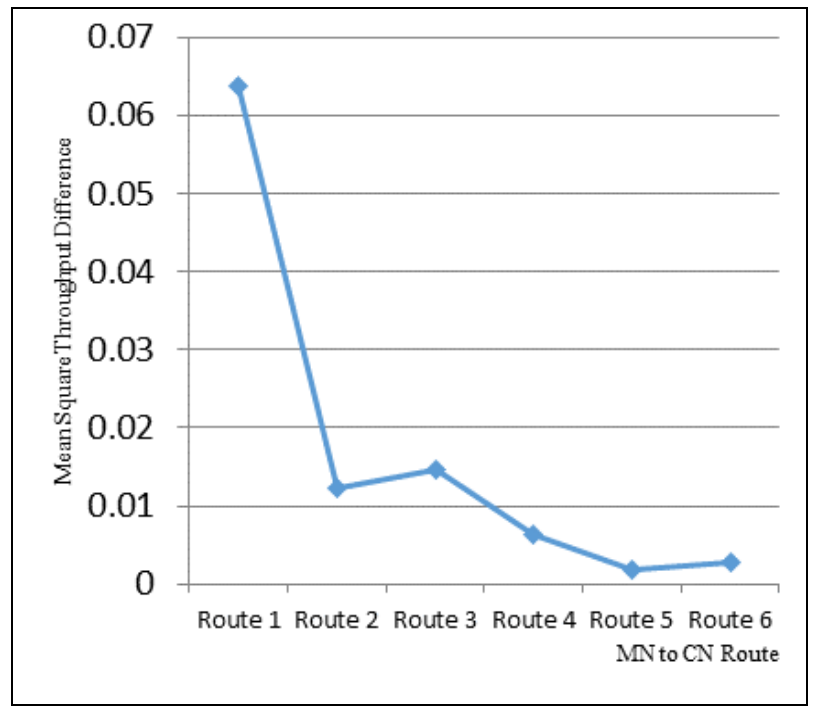

Fig. 8. (b) Mean Square difference in Transmitted Throughput and Received throughput for Routes

Key for Fig. 8

\begin{tabular}{ll}
\hline Route 1 & \multicolumn{1}{c}{$\mathrm{MAG}_{1}-\mathrm{LMA}_{-} \mathrm{MAG}_{5}$} \\
Route 2 & $\mathrm{MAG}_{2}-\mathrm{LMA}-\mathrm{MAG}_{5}$ \\
Route 3 & $\mathrm{MAG}_{3}-\mathrm{LMA}_{-} \mathrm{MAG}_{5}$ \\
Route 4 & $\mathrm{MAG}_{4}-\mathrm{MAG}_{5}$ \\
Route 5 & $\mathrm{MAG}_{5}$ \\
Route 6 & $\mathrm{MAG}_{6}-\mathrm{MAG}_{5}$ \\
\hline
\end{tabular}

Fig. 8. (a) shows a plot of the variations between the transmitting throughput at the $\mathrm{MN}$ and the received throughput at the $\mathrm{CN}$ on the 6 routes existing between the $\mathrm{MN}$ and the $\mathrm{CN}$. The route detection scheme designed becomes operational when $\mathrm{MN}$ is at $\mathrm{MAG}_{4}$. This ensures route $4\left(\mathrm{MAG}_{4}-\mathrm{LAG}_{5}\right)$ is used and the maximum deviation drops to 0.1624 . There is a further drop to 0.0945 when packets use route 5 . Finally route 6 experienced a maximum deviation of 0.1606 . This result shows that deviations of the received throughput from the transmitted throughput were kept to a minimum when the designed scheme selects an optimal route.

The Corresponding MAG ( $\mathrm{MAG}_{5}$ ) has three interfaces connecting it to the LMA and two neighbouring MAGs. This scheme tried to balance the traffic load among these interfaces as shown in Fig 7. Business of the interface is represented by the value one, while a zero represents idleness. Results show that for $47.87 \%$ of the simulated time, packets are received on the LMA interface. $20.62 \%$ of the time it received packets from the $\mathrm{MAG}_{4}$ interface. $19.19 \%$ of the time, $\mathrm{MAG}_{5}$ 's three wired interfaces were idle. The remaining $12.31 \%$ of the time, packets were received on the $\mathrm{MAG}_{6}$ link. This demonstrates an efficient distribution of packets on the interfaces of the $\mathrm{MAG}_{5}$, thereby reducing congestion on all interfaces of $\mathrm{MAG}_{5}$. 


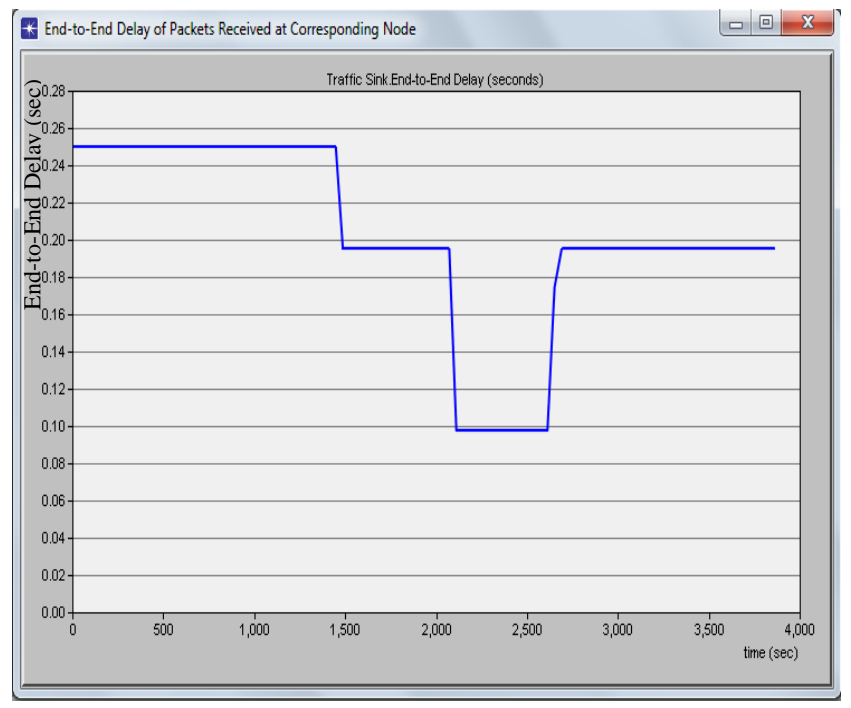

Fig. 9. (a) End-to-End Delay of Packets Received at Corresponding Node (Scenario1)

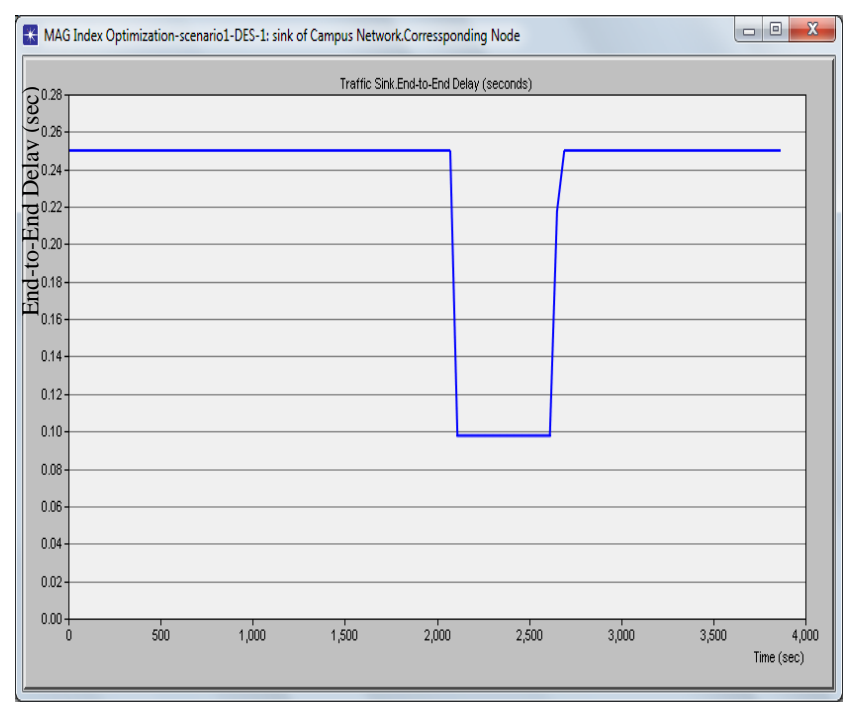

Fig. 9. (b) End-to-End Delay of Packets Received at Corresponding Node (Scenario2)

Fig. 9 (a) shows end-to-end delays of packets received at the corresponding node for Scenario 1. The end-toend delay was calculated by the simulator taking into consideration the signal propagation delay, the transmission delay, the queuing delays and processing delay. The end-to-end delay started at 0.25 seconds, when the Mobile_Node_0 is attached to $\mathrm{MAG}_{1}, \mathrm{MAG}_{2}$ and $\mathrm{MAG}_{3}$ and packets are routed through the route 1 . But the delay drops by $22 \%$ to 0.195 seconds immediately the mobile nodes enters the Region of Coverage (ROC) of $\mathrm{MAG}_{4}$ and the index-based optimization scheme switches packets to the direct link between $\mathrm{MAG}_{4}$ and $\mathrm{MAG}_{5}$. Delays dropped further by $62 \%$ to 0.095 seconds when the Mobile Node enters the ROC of MAG and this is as a result of the fact that both the mobile node and the corresponding node were attached to the same MAG. The delay finally increased back to 0.195 seconds in the ROC of MAG $_{6}$. All of these show a $22 \%$ 
and $62 \%$ drop in the end-to-end delay as a result of the designed scheme over the standard Proxy Mobile IP routing of packets.

Fig. 9. (b) shows end-to-end delay of packets received at the Corresponding Node for scenario 2. Due to more available bandwidth on the MAG-LMA link, delay only drops by $62 \%$ to 0.195 when the mobile node reaches MAG5, as the designed scheme maintains routing through the LMA even when mobile node is at MAG4 and MAG6.

Findings from Network 1 and Network 2 in Fig. 5 (a) and (b) show similar results. This shows that the packet delay encountered was primarily due to queuing delays and processing delays at the LMA and MAGs and not necessarily due to the distance of the links.

\section{Conclusion}

Lack of optimal route has been a problem in the implementation of PIMPv6 network. This work demonstrated the modeling of a locator based route switching scheme to enhance the routing this network. OPNET models were developed for the MAG, LMA, MN and CN. Two network models were used to test the impact of distance between MAG and LMA on packet delays. Two scenarios were used to test the sensitivity of the optimization scheme to bandwidth changes. Results collected include throughput at the MAG, LMA, MN and $\mathrm{CN}$, receiver interface activity level and end-to-end packet delays. Throughput results showed a $28 \%$ drop in differences in throughput values when the designed scheme switches routing to route4. Switching to route5 gave a $41 \%$ drop. The end-to-end results for scenerio1 shows a $22 \%$ (for route 4 and route6) and $62 \%$ (for route5) drop in the end-to-end delay as a result of the designed scheme over the standard Proxy Mobile IP routing of packets. For scenerio 2 the delay only dropped by $62 \%$ to $19.5 \mathrm{~ms}$ when the mobile node reaches $\mathrm{MAG}_{5}$. The $\mathrm{MAG}_{5}$ Interface activity results show that for $47.87 \%$ of the simulated time, packets are received on the LMA interface. $20.62 \%$ of the time it received packets from the $\mathrm{MAG}_{4}$ interface. $19.19 \%$ of the time, $\mathrm{MAG}_{5}$ 's three wired interfaces were idle. The remaining $12.31 \%$ of the time, packets were received on the $\mathrm{MAG}_{6}$ link. This demonstrates an efficient distribution of packets on the interfaces of the $\mathrm{MAG}_{5}$.

\section{References}

[1] Islam, R. U., Mischa, S., and Hans-Joerg, K., "Nomadic mobility between smart homes", pp. 1062 - 1067, 2012. Anaheim, CA, USA.

[2] Yana, B., Mei, S., and Junde, S., "Seamless Mobility Using Mobile IPv6", pp. 1 - 8, 2005, Guangzhou.

[3] Ibrahim, A. S., Mohamed, O., and Borhanuddin, M. A., "Mobility Management for IP-based Next Generation Mobile Networks: Review, Challenge and Perspective", Elsivier Journal of Network and Computer Applications, 35, pp. 295-315, 2012.

[4] Z. Zhenkai, W. Ryuji and Z. Lixia, "A Survey of Mobility Support in the Internet, RFC 6301," 2011. http://datatracker.ietf.org/doc/rfc6301/.

[5] Myung-Kyu, Y., Sung-Yeol, Y., Seok-Cheon, P., and Young-Kyu, Y., "Adaptive Mobility Management Scheme for Proxy Mobile IPv6 Networks with IP Paging Support", 7th International Conference on Signal Image Technology and Internet-Based Systems, 1, pp. 9 - 15, 2011, Dijon.

[6] Gundavelli, S., Leung, K., Devarapalli, V., Chowdhury, K., \& Patil, B., Proxy Mobile IPv6, RFC 5213. Retrieved from datatracker.ietf.org: http://datatracker.ietf.org/doc/rfc5213/, 2008.

[7] Sanjin, J., Myung-Ki, S., and Hyoung-Jun, K., "Implementation of Route Optimization Mechanism Supporting IPv4/IPv6 Transversal in Proxy Mobile IPv6", 11th International Conference on Advanced Communication Technology, 2, pp. 1242 - 1244, 2009, Phoenix Park.

[8] Sanjin, J., \& Myung-Ki, S., "Route Optimization Scheme for Proxy Mobile IPv6 (PMIPv6)", 10th International Conference on Advanced Communication Technology, 2, pp. 1097 - 1100, 2008, GangwonDo. 
[9] Farhana F. L. and Wenbing Y., "Implementation Architecture of Proxy Mobile IPv6 Protocol for NS2 Simulator software", International Conference on Communication Software and Networks, pp. 287-291, 2009, Macau.

[10] Hyon-Young C., Sung-Gi M., Youn-Hee H., Jungsoo P. and Hyoungjun K. "Implementation and Evaluation of Proxy Mobile IPv6 in NS-3 Network Simulator", 5th International Conference on Ubiquitous Information Technologies and Applications, pp. 1 - 6, 2010, Sanya.

[11] Byung-Jin, H., Jae-Min, L., Jong-Hyouk, L., \& Tai-Myoung, C., "PMIPv6 Route Optimization Mechanism using the Routing Table of MAG", 3rd International Conference on Systems and Networks Communications, 1, pp. $274-279$, 2008, Sliema.

[12] Tsunehiko, C., Hidetoshi, Y., Ashutosh, D., Dana, C., \& Henning, S., "Route Optimization for Proxy Mobile IPv6 in IMS Network Network", 2nd International Conference on Signal Processing and Communication Systems, 1, pp. 1 - 9, 2008, Gold Coast, QLD.

[13] Jungwook, S., Heemin, K., \& Sunyoung, H., "Route Optimization in PMIPv6 Environment", 9th IEEE International Conference on Computer and Information Technology, 2, pp. 341 - 346, 2009, Xiamen.

[14] Young-Hyun, C., \& Tai-Myoung, C., "Enhanced Light Weight Route Optimization in Proxy Mobile IPv6", 5th International Conference Joint Conference on INC, IMS and IDC, 1, pp. 501 - 504, 2009, Seoul.

[15] Jin, S. C., Boo, G. J., \& Tae-il, K., "LMA Initiated Route Optimization Protocol for Improving PMIP Handover Performance", Journal of IEEE Communications Letters, 13 (11), 871-873, 2009.

[16] Jianfeng, G., Ilsun, Y., Changqiao, X., Huachun, Z., \& Hongke, Z., "Survey on Route Optimization Schemes for Proxy Mobile IPv6", 6th International Conference on Innovative Mobile and Internet Services in Ubiquitous Computing, pp. 541 - 546, 2012, Palermo.

\section{Author(s) Profile}

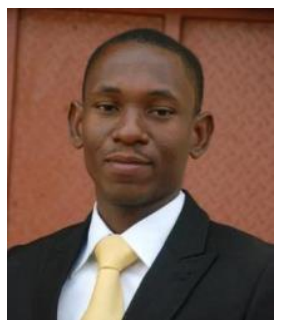

Michael Okwori is currently an Assistant Lecturer at the Department of Telecommunications Engineering, Federal University of Technology, Minna, Nigeria where he just obtained his Masters of Engineering Degree in Communications Engineering. He obtained a First Class Honours Bachelor of Engineering Degree from the Department of Electrical and Computer Engineering, Federal University of Technology Minna in 2007. His Research interests include Mobility Management in IP Networks, Mobile Telecommunication Architecture, Optical Communications and Wireless Sensor Networks.

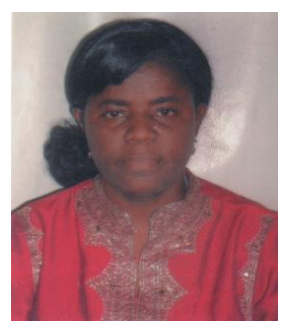

Elizabeth N. Onwuka obtained a Bachelor of Engineering (B.Eng.) Degree from Electrical and Computer Engineering Department, Federal University of Technology (FUT) Minna, Niger State, Nigeria, in October 1992; a Master of Engineering (M.Eng.) Degree, in Telecommunications, from Electrical and Computer Engineering Department, FUT, Minna, Niger State, Nigeria, in March 1998; and Doctor of Philosophy (PhD) Degree, in Communications and Information Systems Engineering, from Tsinghua University, Beijing, People's Republic of China, in June 2004. She is currently an associate professor in the Department of Telecommunications Engineering FUT, Minna, Niger state. Her research interest includes Mobile communications network architecture, IP networks, handoff management, paging, network integration, and resource management in wireless networks, wireless sensor networks. 


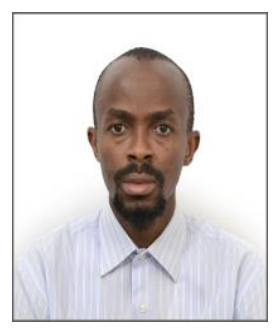

Abiodun Musa AIBINU is presently with the Department of Mechatronics Engineering, Schhol of Engineering and Engineering Technology, Federal University of Technology, Minna, Nigeria. His research interests are: Biomedical Signal Processing; Digital Image Processing; Instrumentation and Measurements; Telecommunication system Design and Digital System Design

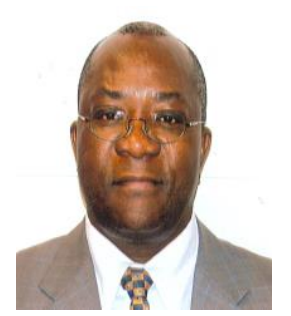

Professor Okechukwu C. Ugweje is currently the Vice President, Academic and Student Affairs, at the Digital Bridge Institute (DBI), Abuja. He also holds appointments as an Adjunct Professor of Electronic Engineering at the University of Nigeria, Nsukka, and Visiting Professor at the Federal University of Technology, Minna. Before he joined DBI, he was a tenured Professor of Electrical and Computer Engineering at The University of Akron, Akron $\mathrm{OH}$, USA teaching courses in Information and Communication Technologies (ICT), Digital Signal Processing, Probability and Random Processes, Computer Networking and Architecture. His consulting, teaching and research interests are in all areas of ICT, terrestrial wireless and satellite communication networks. Prior to the above appointments, he worked as a telecommunications engineer with Siemens Network Telecom Inc., Boca Raton, Florida. Prof. Ugweje is a member of Tau Beta Pi, Eta Kappa Nu, and several IEEE societies He is married with children

How to cite this paper: M. Okwori, E. N. Onwuka, A. M. Aibinu, O. C. Ugweje,"Implementation of a Locator-Based Route Switching Scheme for Improved Routing in Proxy Mobile IPv6", IJWMT, vol.4, no.4, pp.1-19, 2014.DOI: 10.5815/ijwmt.2014.04.01 